their patients' use of internet as part of routine history taking. Internet \& ICT use is like the two faces of the coin, with both their positive or health threatening aspects. Among the positive features, one should cite an easy access to educational and health information, the promotion of socialization enhancing activities-including those directed at youngsters with chronic conditions -, opportunities for leisure activities which can be quite creative. Isolation, health symptoms, exposure to violence \& pornography, incitement to delinquent behavior or self-harm all belong to the darker side of the coin. The assessment of internet use and of situations of problematic use of ICT should not be restricted to the number of hours spent on the computer but should also tackle the impact of the use on the adolescent's school and social life, as well as the nature of the relation between the young person and ICT: adolescents who use internet for social contacts, to build new worlds or to learn are much less at risk then those who are on line gamers or/and sensation seekers. The presentation will close with some universally exploitable advices for parents.

\section{BINGE DRINKING: NOT AN INNOCENT PROBLEM}

doi:10.1136/archdischild-2012-302724.0039

1J De Dooy, ${ }^{2} \mathrm{G}$ Van Hal, ${ }^{3} \mathrm{G}$ Van Laecke, ${ }^{4} \mathrm{~J}$ Ramet. 'Paediatric Intensive Care, University of Antwerp, Antwerp University Hospital, Edegem; '2Epidemiology and Social Medicine; ${ }^{3}$ Faculty of Medicine, University of Antwerp, Antwerpen; ${ }^{4}$ Paediatrics, University of Antwerp, Antwerp University Hospital, Edegem, Belgium

More and more paediatricians are confronted with teen drinking and the problems that result from it. A new trend is the so called "binge-drinking". This means consumption of excessive amounts of alcohol in a very short period of time with the goal of getting drunk as fast as possible.

Within 1 hour after ingestion, alcohol is absorbed in the stomach and small intestine. Toxic effects of alcohol can occur from a plasma concentration of $0.5-1 \%$ o $(=500-1000 \mathrm{mg} / \mathrm{l})$ or an intake of 600 $\mathrm{mg} / \mathrm{kg}$ alcohol. This is equal to ingestion of $120 \mathrm{ml}$ liquor (alcohol concentration $30 \%$ ) in an adolescent of $40 \mathrm{~kg}$. The effects are caused by a suppression of the central nervous system and they occur faster in younger people than in adults due to less extracellular volume. Also individual factors like gender, use of other medications or drugs and drinking habits play a role. All this factors can result in a broad spectrum of symptoms (relaxation, altered perception of the environment, prolonged reaction time, amnesia, nausea, vomiting and in more severe cases respiratory depression, coma and death).

When a child with possible alcohol intoxication is admitted to the emergency department, general "APLS" guidelines (Advanced Pediatric Life Support) should be followed.

After the "acute" event of the alcohol intoxication, multidisciplinary follow-up of the patient is very important.

In Belgium, no objective data exist on the problem of binge drinking whilst among paediatricians there is great concern about it. Therefore, we will conduct a national survey on this topic.

\section{UPDATE ON TUBERCULOSIS FOR THE GENERAL PAEDIATRICIAN}

doi:10.1136/archdischild-2012-302724.0040

MJ Mellado. Pediatric Infectious and Tropical Diseases, Hospital Carlos III. Servicio de Pediatria, Madrid, Spain

About one million of TB cases by year still occur in children. TB childhood diagnosis is an urgent task and even suspected TB disease should also be treated. Clinical features; thorax-X-ray; TST; smear/ culture/PCR from gastric aspirated-induced sputum are diagnosis tools.
Children Key-recommendations:

1. Anti-TB drugs new doses in children, supported by pharmacokinetic (WHO):

Isoniazid (H) $10 \mathrm{mg} / \mathrm{kg}(10-15) \mathrm{max} .300 \mathrm{mg} /$ day

Rifampicin (R) 15 (10-20) 600

Pyrazinamide (Z) 35 (30-40) 2000

Ethtambutol (E) 20 (15-25) 2500

2. All children have to be included in one of:

Exposure or Latent-TB-infection, or TB disease; because need different management. Although children, usually not been infectious, family prophylaxis interrupts disease's dissemination.

3. TB management:

1 TB exposure: H 2 months; repeat TST, if positive action as LTBI,

2 LTB infection: $\mathrm{H}$ 6-9 months or HR 3 months,

3 TB disease:

Children living in high-HIV-prevalence or high-H-resistance area, with pulmonary/lymphadenitis TB; or children with extensive pulmonary disease in low-HIV-prevalence o low-H-resistance area, should be treated: 2 months HRZE +4 months HR. - In meningitis TB: 2HRZE + $10 \mathrm{HR}$. - HIV-negative children and low-HIV-prevalence and low-H-resistance area, could be treated: $2 \mathrm{HRZ}+4 \mathrm{HR}$. Maintenance period: thrice-weekly regimens can be considered, only if well established Directly Observed Therapy. HIV-infected children or living in HIV-high-prevalence area should not be treated with intermittent regimens. - Streptomycin should not be used as a part of firs-line regimen in pulmonary/lymphadenitis TB. Children with TB-MDR should be treated: fluoroquinolones + aminoglucoside guide by an expert.

\section{PEDIATRIC TUBERCULOSIS IN TWO TERTIARY HOSPITALS IN ROME: A 20-YEAR RETROSPECTIVE STUDY}

doi:10.1136/archdischild-2012-302724.0041

'D Buonsenso, ${ }^{2} \mathrm{~L}$ Lancella, ${ }^{1} \mathrm{P}$ Valentini. ${ }^{1}$ Catholic University of the Sacred Heart, ${ }^{2}$ Bambino Gesù Children's Hospital, Rome, Italy

Background and aims Tuberculosis is among the top 10 causes of child death worldwide. We aimed to describe epidemiological, clini$\mathrm{cal}$ and microbiological features of patients with active tuberculosis admitted in two tertiary hospitals in Rome.

Methods Retrospective study of patients $<16$-year-old evaluated between 1990 and 2009.

Results 214 cases of active tuberculosis were identified (132 definite, 82 probable). Pulmonary involvement was the most common form $(75.5 \%)$, followed by lymphadenopathy (15.4\%) and central nervous system tuberculosis (11\%). Fever $(51.86 \%)$ and cough (40\%) were the most common presenting symptoms. $23.4 \%$ children were asymptomatic on admission. Sensitivities for Tuberculin Skin Test and Quantiferon test were $93.4 \%$ and $97 \%$ respectively. Both tests were performed in 52 children agreeing in 49 cases (94\%). Sensitivities for culture, Ziehl-Neelsen staining and polymerase chain reaction were $58 \%, 25 \%$ and $66.3 \%$ respectively. The adult source case was identified in $28 \%$ cases. History of contact with a patient with active tuberculosis was associated with pulmonary tuberculosis $(\mathrm{P}=0.0014)$, while negative history of contact was associated with lymph nodal $(\mathrm{P}=0.0064)$ and central nervous system tuberculosis $(\mathrm{P}=0.05)$.

Conclusions Our study emphasize the difficulty in managing children with suspected tuberculosis, since the absence of constitutional symptoms cannot exclude tuberculosis and bacteriological confirmation is the exception. Immunological diagnosis can be valuable tool to identify tuberculosis infected children since quantiferon 
showed high sensitivity in all age-groups; this is of primary importance since early identification of children with latent tuberculosis infection and appropriate chemoprophylaxis represent the most important tool to reduce tuberculosis burden.

\section{ANTIBIOTIC USE IN INFANTS IN THE FIRST YEAR OF LIFE IN FIVE EUROPEAN COUNTRIES}

doi:10.1136/archdischild-2012-302724.0042

M van Stuijvenberg, J Stam, P Sauer, for the MIPS 1 Study Group. Pediatrics, Beatrix Childrens Hospital UMCG, Groningen, The Netherlands

Background and aims In view of antibiotic resistance problems and development of atopic diseases there is a need to improve the appropriateness of antibiotic use, especially in young children. Antibiotics are mostly prescribed for upper respiratory tract infections (URTI) and otitis media (OM), while these are mostly of viral origin. We report antibiotic use for these infections in otherwise healthy term infants up to one year of age.

Methods The study was part of a multicenter nutritional intervention study (DRKS00000201) in which the parents recorded illness symptoms and antibiotic use of their child in a diary. Logistic regression was used to analyse differences in antibiotic use between the participating countries (Italy, Netherlands, Austria, Switzerland, and Germany).

Results The study was completed by 839 children; 4798 illness episodes were reported, of which 501 (10.4\%, range 3.9-18.4\%) were treated with antibiotics.

URTI occurred in 2855 (59\%) episodes (range 55-64\%). Antibiotics were used more often in Italy compared to Switzerland: $18.8 \%$ versus $1.4 \%, \mathrm{OR}=0.06(\mathrm{CI} 95 \%=0.02-0.1)$.

OM occurred in 184 (3.8\%) episodes (range 2.0-6.8\%). Antibiotics were used more often in Italy compared to the Netherlands: $82 \%$ versus $55 \%, \mathrm{OR}=0.3(\mathrm{CI} 95 \%=0.1-0.6)$.

Conclusions Antibiotic use varies significantly between European countries while the occurrence of URTI and OM is rather similar. In the development of methods to increase appropriate use of antibiotics other factors, such as physicians attitude, parental influence, and other socio-economic determinants may better be taken into account.

\section{EVALUATION OF THE EFFICACY OF L-ISOLEUCINE SUPPLEMENTED FOOD AND VITAMIN D IN THE TREATMENT OF ACUTE DIARRHEA IN CHILDREN}

doi:10.1136/archdischild-2012-302724.0043

NH Alam, H Ashraf. Centre for Nutrition and Food Security (CNFS), International Centre for Diarrhoeal Disease Research, Bangladesh (ICDDR, B), Dhaka, Bangladesh

Introduction Antimicrobial peptides represent an important component of the innate defense of organisms and have activities against all microbes. Recently, L-isoleucine and Vitamin D have been found to induce antimicrobial peptides. Therefore, L-isoleucine and vitamin $\mathrm{D}$ might have therapeutic potentials in the management of infectious diarrhea.

Objectives The objectives of this study were to examine if addition of L-isoleucine and/or vitamin $\mathrm{D}$ to a diet reduces the stool weight and/or duration of acute diarrhea in children.

Methods This was a double blind randomized clinical trial in 107 children aged 6 to 36 months attending the ICDDRB hospital with acute diarrhea: 28 children received:

a. L-isoleucine $(2 \mathrm{~g} / \mathrm{d})$ added to milk suji

b. 27 received Vitamin D 1000 IU/d added to Milk suji;

c. $26 \mathrm{~L}$-isoleucine $(2 \mathrm{~g} / \mathrm{d})$ plus vitamin D $1000 \mathrm{IU} / \mathrm{d}$;

4. 26 Milk suji without L-isoleucine and vitamin $\mathrm{D}$.
Other managements were similar in all groups. Stool weight(g) and duration of diarrhea were the primary outcomes.

Results There was a trend in stool weight reduction in the groups receiving $\mathrm{L}$-isoleucine and the reduction was significant on day 2 (mean $\pm \mathrm{SD}$, L-isoleucine vs. vit $\mathrm{D}$ vs. $\mathrm{L}$-isoleucine + vit $\mathrm{D}$ vs. control, $276 \pm 228$ vs. $386 \pm 302$ vs. $301 \pm 181$ vs. $447 \pm 325, p=0.039)$ and day3 ( $176 \pm 157$ vs. $321 \pm 273$ vs. $276 \pm 169$ vs. $341 \pm 292, p=0.045)$. The duration of diarrhea was similar in all groups.

Conclusion L-isoleucine supplemented food reduces stool weight in children with acute diarrhea.

\section{COMPARISON OF THE EFFICACY OF C-REACTIVE PROTEIN, PROCALCITONIN, INTERLEUKIN-6 LEVELS AND NEW LEUKOCYTE PARAMETERS IN THE DIAGNOSIS OF NEONATAL SEPSIS}

doi:10.1136/archdischild-2012-302724.0044

'T Çelik, ${ }^{2} 0$ Portakal, 'Ş Yiğit, ${ }^{3} \mathrm{G}$ Hasçelik, ${ }^{1} \mathrm{~A}$ Korkmaz, ${ }^{1} \mathrm{M}$ Yurdakök. 'Department of Pediatrics, Division of Neonatology, Hacettepe University, Faculty of Medicine; ${ }^{2}$ Department of Biochemistry; ${ }^{3}$ Department of Microbiology, Hacetepe University, Faculty of Medicine, Ankara, Turkey

Background and aims Sepsis is an important cause of morbidity and mortality among newborn infants. Blood culture is the gold standard. Early and definitive diagnosis of neonatal sepsis is difficult because its sings and symptoms are nonspecific. New leukocyte parameters such as neutrophil volume (MNV), conductivity (MNC), scattering (MNS) and volume distribution width (NDW) were introduced in the diagnosis of sepsis recently. We aimed to investigate these parameters in newborn sepsis and compare their efficacy with serum CRP, Procalcitonin (PCT), IL-6 levels.

Methods This study was conducted in Hacettepe University Neonatology Unit, between July 2010 and February 2012. Total 227 newborns, 116 sepsis (40 proven, 76 clinical sepsis) and 111 control included in the study.

Results Results are summarized in the Table 1.

Abstract 44 Table 1

\begin{tabular}{lcccccc}
\hline & $\begin{array}{c}\text { Cut-off } \\
\text { levels }\end{array}$ & $\begin{array}{c}\text { Sensitivity } \\
(\%)\end{array}$ & $\begin{array}{c}\text { Specificity } \\
(\%)\end{array}$ & PPV (\%) & NPV (\%) & AUC (\%) \\
\hline CRP (mg/dl) & $>0.16$ & 75.0 & 76.3 & 50.8 & 91.9 & 77.7 \\
PCT (ng/dl) & $>0.44$ & 75.0 & 86.0 & 60.4 & 89.3 & 86.9 \\
IL-6 (pg/ml) & $>15.40$ & 70.8 & 74.2 & 45.5 & 91.0 & 72.5 \\
I/T ratio & $>0.19$ & 62.5 & 92.5 & 79.4 & 88.9 & 81.5 \\
MNV (au) & $>159.50$ & 37.5 & 94.6 & 71.4 & 80.8 & 63.4 \\
MNC (au) & $<144.50$ & 78.4 & 46.8 & 35.2 & 86.7 & 62.6 \\
MNS (au) & $<141.50$ & 86.5 & 37.6 & 32.7 & 87.2 & 64.5 \\
NDW (au) & $>29.25$ & 66.7 & 75.3 & 43.9 & 84.0 & 68.2 \\
\hline
\end{tabular}

Sensitivity, specificity, positive and ne

Conclusions In conclusion new CBC parameters can be helpful in differential diagnosis of newborn sepsis in addition to other screening parameters. MNV seems the most useful parameter with the highest spesifity.

\section{EFFECT OF A MULTIMODAL INTERVENTION PROGRAM TO PREVENT OBESITY IN EARLY CHILDHOOD}

doi:10.1136/archdischild-2012-302724.0045

${ }^{1} \mathrm{HM}$ Donkor, 'JH Grundt, 'J Hurum, 'ABK Sundby, ${ }^{2} \mathrm{~T}$ Skundberg, ${ }^{3,4} \mathrm{~T}$ Markestad. ${ }^{1}$ Department of Paediatrics, Innlandet Hospital Trust, Lillehammer Hospital, Lillehammer, ${ }^{2}$ Department of Paediatrics, Innlandet Hospital Trust, Gjövik Hospital, Gjövik; ${ }^{3}$ Research Division, Innlandet Hospital Trust, Brumunddal; " 4 Department of Clinical Medicine, University of Bergen, Bergen, Norway 